\title{
Towards Intra-operative PET for Head and Neck Cancer: Lymph Node Localization Using High-Energy Probes
}

\author{
Dzhoshkun I. Shakir ${ }^{1,2}$, Aslı Okur ${ }^{1,2}$, Alexander Hartl ${ }^{1,2}$, Philipp Matthies ${ }^{1}$, \\ Sibylle I. Ziegler ${ }^{2}$, Markus Essler ${ }^{2}$, Tobias Lasser ${ }^{1,3}$, and Nassir Navab ${ }^{1}$ \\ 1 Computer Aided Medical Procedures (CAMP), \\ Technische Universität München, Germany \\ 2 Department of Nuclear Medicine, Klinikum rechts der Isar, \\ Technische Universität München, Germany \\ 3 Institute of Biomathematics and Biometry, HelmholtzZentrum München, Germany
}

\begin{abstract}
We present a novel approach for intra-operative localization of lymph nodes and metastases in the head and neck region using the radio-tracer $\left[{ }^{18} \mathrm{~F}\right] \mathrm{FDG}$. By combining an optical tracking system with a high-energy gamma probe to detect $511 \mathrm{keV}$ annihilation gammas, we enable intra-operative PET to visualize activity distributions. Detection of these gammas is modeled ad-hoc analytically, taking into account several factors affecting the detection process. This allows us to iteratively reconstruct the radio-tracer distribution within a localized volume of interest. As a feasibility study we analyze clinical data of 7 patients with tumors in the head and neck region, and derive a realistic neck phantom configuration with $\left[{ }^{18} \mathrm{~F}\right]$ FDG-filled lesions mimicking tumors and lymph nodes. We demonstrate the capabilities and limitations of our approach using that neck phantom. We also outline possible improvements to make our method clinically viable towards less invasive surgeries.
\end{abstract}

\section{Introduction}

Head and neck squamous (epithelial) cell carcinoma (HNSCC) is diagnosed in 500,000 patients each year. HNSCC primarily affects the oropharynx, oral cavity hypopharynx and larynx 1, but has a risk of metastasizing into the cervical lymph nodes (LNs). It is therefore important for prognosis to assess the status of LNs in this region and remove the ones containing metastases. In the current clinical workflow, sonography, MRI, Computed tomography (CT) or $\left.{ }^{18} \mathrm{~F}\right] \mathrm{FDG}-$ Positron-Emission-Tomography ( $\left[{ }^{18} \mathrm{~F}\right]$ FDG-PET - referred to as simply PET from this point on) are used for pre-operative LN staging in HNSCC patients. Compared to others, PET was found to detect cervical LN metastases with the highest sensitivity and specificity [2. The aim of surgical treatment is to completely resect the tumor and all LN metastases. However, the intra-operative localization of metastatic LNs only is difficult, so in practice all LNs in the vicinity of the suspicious one(s) are resected by a neck dissection procedure. On the other hand, the high post-operative morbidity risk due to the presence of 
vital anatomic structures is an indication for minimizing invasiveness in head and neck (HN) interventions. Aesthetic motivations are also obvious. Thus it is of high clinical relevance to intra-operatively guide the surgeon to selectively resect the few PET-positive metastatic LNs, especially when critical structures such as nerves or vessels make resection difficult.

In this work we describe freehand PET (fhPET), a novel system for the intraoperative detection of metastatic LNs with an increased glucose metabolism. fhPET allows to intra-operatively image the region containing the PET-positive LNs. Our system combines a high-energy gamma probe (HE probe) (that can detect the $511 \mathrm{keV}$ gammas released upon the annihilation of the positrons emitted by $\left[{ }^{18} \mathrm{~F}\right] \mathrm{FDG}$ with electrons) with a 3D optical tracking system, similar to the freehand SPECT (fhSPECT) system mentioned in 34. The detection physics are modeled with an analytical ad-hoc model that allows for iterative reconstruction of the $3 \mathrm{D}$ radio-tracer distribution within the volume of interest (VOI). In contrast to freehand SPECT, this technique has to deal with highenergy $(511 \mathrm{keV})$ gamma rays (compared to the $140 \mathrm{keV}$ gamma rays of ${ }^{99 \mathrm{~m}} \mathrm{Tc}$ ).

Most related work uses $\mathrm{HE}$ probes without tracking and navigation support; for instance in HNSCC [5] and thyroid cancer applications [6]. There is work on dedicated intra-operative PET scanners 78 , combined intra-operative PET and trans-rectal ultrasound [9], or handheld PET imaging probes with an external detector ring for full tomographic data [10. A more suitable approach for the operating room (OR) is outlined in [11,12.

\section{Materials and Methods}

\subsection{High-Energy Gamma Probes (HE Probes)}

A HE probe is a pen-sized, hand-held device, typically with a big shielding head around the detector (see fig. 1(a), that can detect annihilation gamma rays. The annihilation takes place within a very limited distance from the emitting atom (typically below $2 \mathrm{~mm} \mathrm{[13])} \mathrm{and} \mathrm{results} \mathrm{in} \mathrm{two} 511 \mathrm{keV}$ gamma rays in opposite directions to each other. PET systems are based on hardware that can detect such simultaneous gamma rays (coincidences). A HE probe does not detect coincidences, but only single $511 \mathrm{keV}$ gamma rays.

\subsection{Tomographic Imaging with 1D HE Probes}

To generate tomographic images from the HE probe data we need to combine it with a spatial localization system, such that the VOI can be scanned with the probe while the probe signals are recorded synchronously to the probe positions with respect to the VOI. This VOI is discretized into voxels and we decompose each measurement $m_{j}$ into a linear combination of the contributions $a_{i j}$ of the unknown activity values $x_{i}$ in all voxels:

$$
m_{j}=\sum_{i} a_{i j} x_{i}
$$




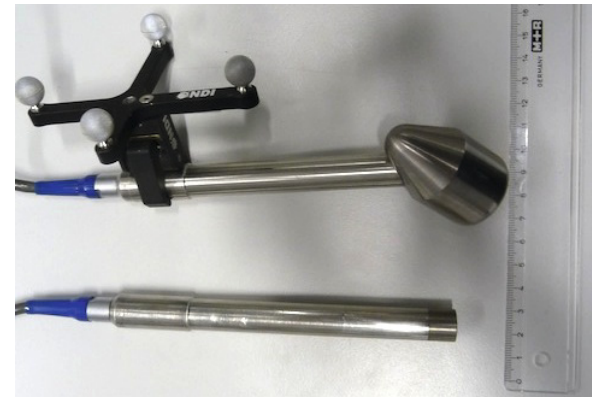

(a)

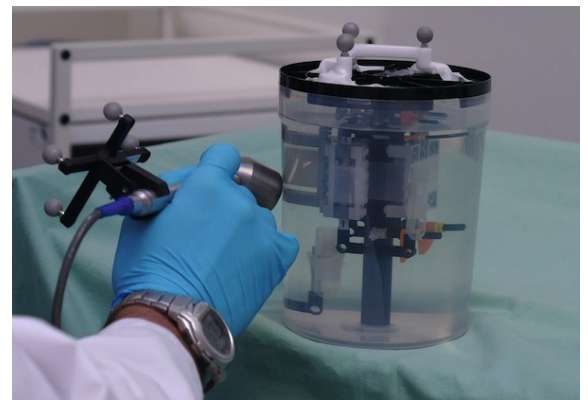

(b)

Fig. 1. (a) top: HE probe, bottom: low-energy gamma probe. (b) Neck phantom.

Using this decomposition, all the measurements within a scan can be stacked into a system of linear equations: $m=A x$. By inverting this system we can retrieve the activity distribution in our VOI using a solver like MLEM (Maximum Likelihood Expectation Maximization) [3]. However, for the inversion, the system matrix $A$ with the contributions $a_{i j}$ is needed. As we do not have a fixed acquisition geometry we need to compute the contributions on the fly using an ad-hoc model of the detection physics of our probe.

\subsection{Ad-Hoc Model of Detection Physics}

We model the physical factors affecting the detection of gamma rays analytically, based on the known geometric properties of our HE probe. This is our ad-hoc model of detection physics.

First of all, our model computes the geometric attenuation, which determines the portion of the initial radiation that should in the ideal case reach the detector due to the isotropy of radiation. This is computed with the solid angle $\Omega$ subtended by the detector of the probe on a point source.

In the next step, the effects of the shielding and the absorption in the detector of the probe are computed using the mean lengths that gamma rays will traverse through the shielding and the detector. These lengths are obtained by dividing the space around the probe into partitions in each of which these lengths can be computed with a unique formula. Due to symmetry the computations can be reduced to a profile slice through the probe. In this slice we consider the four rays that reach the four corners of the detector and for each of these rays we compute the length of interaction $l_{i}$ that the ray traverses through the detector (see fig. 2(a)). The probability of an interaction in the detector along this ray can be computed using the formula $p=1-e^{\mu l_{i}}$, with $\mu$ being a material coefficient (detector: BGO, shielding: tungsten 14]). Using this probability function we can now compute the mean probability $\bar{p}$ of an interaction between two successive rays by integrating over the probability function with the two rays as boundaries and then dividing by the difference in the length of interaction of both rays: 

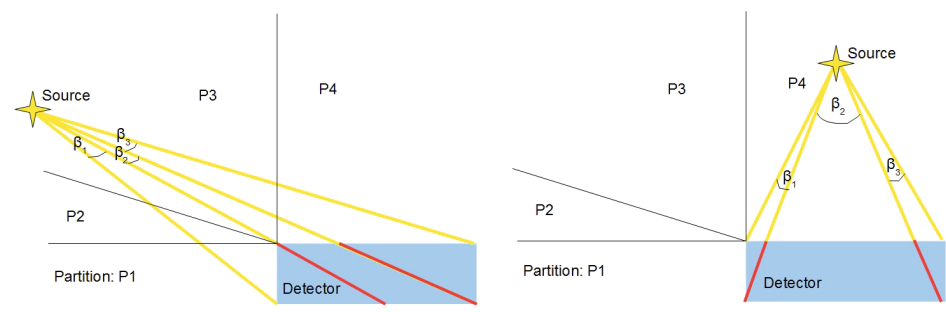

(a)
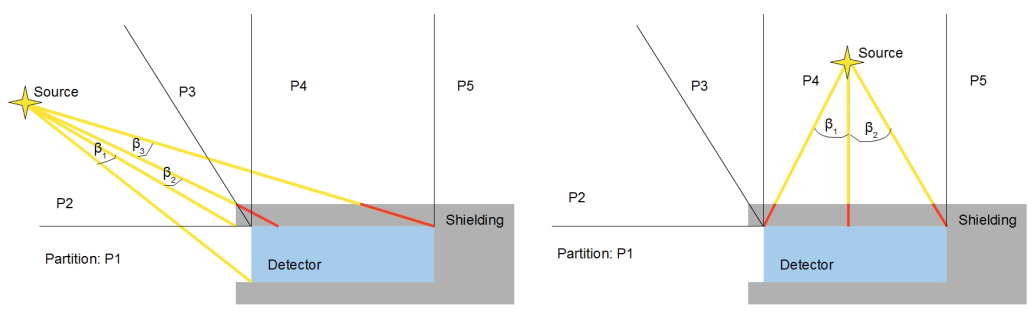

(b)

Fig. 2. The length rays take through the detector (a) and through the shielding (b) are computed depending on the partition $(\mathrm{P} 1, \mathrm{P} 2 \ldots)$ in which the source lies. This is done by using several example rays between which this length (red) changes smoothly. For these rays the probability of an interaction is computed and by integrating between two successive rays. This is then weighted with the angle $\beta_{i}$ between the two rays divided by the sum of all angles, so we get the mean probability for an interaction in the detector/shielding from a specific source position.

$$
\bar{p}=\frac{\int_{l_{i_{n}}}^{l_{i_{n}+1}}\left[1-e^{\mu l_{i}}\right] d l_{i}}{\left|l_{i_{n}}-l_{i_{n+1}}\right|}=\frac{1-\left|e^{\mu l_{i_{n+1}}}-e^{\mu l_{i_{n}}}\right|}{\left|l_{i_{n}}-l_{i_{n+1}}\right|}
$$

By weighting these probabilities with the angle $\beta_{i}$ between these rays and dividing by the total angle between the two outer rays we get the mean probability for an interaction of a gamma ray emitted by a point source on a specific position relative to the probe. Absorption in the shielding is computed in a similar way (see fig. 2(b).

The mean probability for an absorption in the shielding $\overline{p_{s}}$ and in the detector $\overline{p_{d}}$ are then used to compute the amount of radiation that is detected:

$$
a=\left(1-\overline{p_{s}} * \overline{p_{d}} * \frac{\Omega}{4 \pi}\right)
$$

\subsection{System Setup and Challenges}

Our system combines a 1D HE probe (NodeSeeker 800, Intra Medical Imaging LLC, CA, USA) with a 3D (6DOF) optical tracking system (Polaris Vicra, Northern Digital Incorporated, ON, Canada). The combined data gets synchronized by the software running on the application workstation (CSS300, SurgicEye 
GmbH, Munich, Germany). We tuned the software on this workstation to accommodate the described ad-hoc model. Data acquisition and reconstruction are performed in real-time using the workstation (about $5 \mathrm{~min}$ for data acquisition and $1 \mathrm{~min}$ for reconstruction). It also provides an augmented-reality visualization.

Our system is similar in terms of hardware to the fhSPECT technology. However, the accurate modeling of the high-energy gamma rays in matter and their detection in the detector poses additional challenges. One big problem is that due to their high energy levels, these $511 \mathrm{keV}$ gamma rays can penetrate through matter more than e.g. the $140 \mathrm{keV}$ gamma rays of ${ }^{99 m} \mathrm{Tc}$. Thus, HE probes require much thicker shielding, which can still not stop all the gammas. Another problem is the background radiation due to the partially unspecific $\left.{ }^{18} \mathrm{~F}\right] \mathrm{FDG}$ uptake (e.g. muscle activity, inflammation - see fig. 3(a) , contrary to e.g. ${ }^{99 m} \mathrm{Tc}-$ marked tracers used in sentinel LN procedures, where there is almost no unspecific uptake.

\section{$3 \quad$ Experiments}

We conducted three different sets of experiments to evaluate fhPET for the localization of tumors and LNs/metastases in HNSCC. For these, we prepared a phantom simulating a tumor mass and a LN in the neck region, using a plastic box and three plastic lab reservoirs $(2 \mathrm{ml}$ each) (see fig. 1(b)). The reservoirs were attached rigidly and reproducibly to the construction. For the first set of experiments, we evaluated PET/CT images from 7 HNSCC patients (mean age: 53 year, $6 \mathrm{~m} / 1 \mathrm{f}$ ). Each patient had one or two PET-positive LNs. Following the surgical resection, the LNs were histologically examined for metastases and 5 cases were positive. Using these data sets, we calculated the values seen in table 1 we used for injecting $\left[{ }^{18} \mathrm{~F}\right] \mathrm{FDG}$ into the reservoirs (simulating the tumor and the LN). The rest of the phantom was filled with water and with background (BG) activity respectively (simulating cases with no BG and unspecific BG).

Table 1. The geometrical and activity-related parameters obtained from patient data

\begin{tabular}{|l|c|}
\hline tumor depth from the surface: & $5.3 \pm 1.06 \mathrm{~cm}$ \\
lymph node depth from the surface: & $2.6 \pm 0.99 \mathrm{~cm}$ \\
\hline tumor-to-background (T/BG) uptake ratio: & $3.3 \pm 1.17$ \\
tumor-to-lymph node (T/LN) uptake ratio: & $1.5 \pm 0.97$ \\
\hline tumor uptake: & $23.8 \pm 9.41 \mathrm{kBq} / \mathrm{ml}$ \\
lymph node uptake: & $21.3 \pm 12.75 \mathrm{kBq} / \mathrm{ml}$ \\
\hline
\end{tabular}

In the second set of experiments, we used higher activity, which we also varied, in order to be able to assess the boundary conditions of our system, i.e. from which activity level on we can actually see the tumor and the LN. 


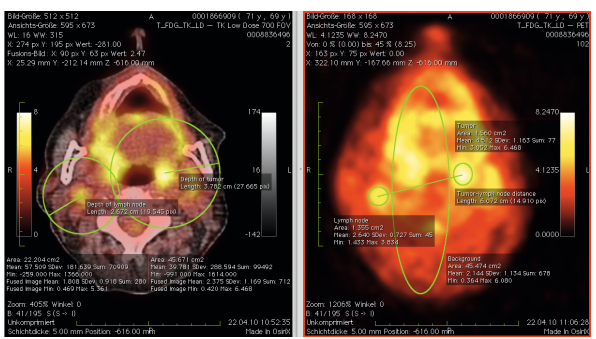

(a)

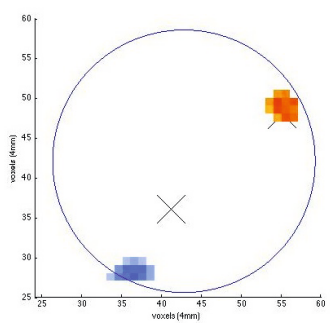

(b)

Fig. 3. (a) The tumor and LN locations, as well as depths, were identified in the overlaid PET/CT images (left). The corresponding average activity values were obtained from PET images (right). In addition, the unspecific background radiation values were calculated (in this case the large elliptic region).(b) A transverse slice of one reconstructed image. Blue blob: reconstructed tumor (the cross nearby showing the tumor location in the ground truth). Red figure: reconstructed LN (the cross within showing the LN location in the ground truth). Circle: an outline of the phantom.

In the third set of experiments, we used the same activity ratios as in the second set, but this time we used ${ }^{99 m}$ Tc. Our aim here was to compare the images obtained with the fhSPECT system and our fhPET system, in the light of the discussion about the additional challenges of fhPET within sec. 2.4

Two operators scanned each phantom configuration two or three times respectively, each time covering about 120 degrees around the phantom, and obtaining 3000 measurement points. In each case the resulting system of linear equations was inverted using MLEM with 20 iterations. The obtained reconstruction was smoothed using a 4 or $6 \mathrm{~mm}$ Gaussian filter to reduce reconstruction noise due to the highly under-sampled acquisition with insufficient statistics.

\section{Evaluation and Results}

For qualitative evaluation, we checked the visibility of the tumor and the LN in the reconstructions. Within the first set of scans (realistic activity concentrations) we were able to identify the $\mathrm{LN}$ in 3 (none with BG activity) of the 7 reconstructions. We could identify the tumor in none of the 7 reconstructions, as it was seated much deeper than the LN.

In the second set of experiments we were able to identify the LN in all of the 17 reconstructions. Moreover, we were able to see the tumor in 6 (3 of those with BG activity) reconstructions (for example see fig. $3(\mathrm{~b})$. In one of these we could even distinguish the two reservoirs next to each other, simulating the tumor.

We further obtained a CT image of our phantom to serve as a ground truth. Using the phantom tracking target seen in fig. 1(b) (already visible in the CT), we registered the $\mathrm{CT}$ image to the fhPET images. In the CT images, we manually selected the midpoint of the LN and the tumor. Using the registration, we computed the distance between the centroid of the LN in the fhPET images and 
Table 2. Different phantom configurations and accuracies achieved with fhPET

\begin{tabular}{|l|c|c|c|}
\hline Experiment setup & BG:T:L & Lymph node loc. error $(\mathrm{mm})$ & Tumor loc. error $(\mathrm{mm})$ \\
\hline 1. $\left[{ }^{18} \mathrm{~F}\right]$ FDG low & $0: 17: 10$ & $12.67 \pm 2.48$ & NA \\
\hline 2. $\left[{ }^{18} \mathrm{~F}\right]$ FDG high & $0: 20: 20$ & $13.57 \pm 4.22$ & $34.79 \pm 6.20$ \\
& $1: 20: 20$ & $14.41 \pm 5.75$ & $47.39 \pm 22.28$ \\
\hline $3 .{ }^{99 m}$ Tc high & $0: 20: 20$ & $11.35 \pm 4.26$ & $39.39 \pm 10.60$ \\
& $1: 20: 20$ & $10.85 \pm 4.07$ & $20.42 \pm 2.55$ \\
\hline
\end{tabular}

its midpoint in the CT. We did the same for the tumor as well, for the cases where the tumor was visible in the fhPET images.

\section{Discussion and Conclusion}

The results show the ability to reconstruct the mock LN in all cases, with a localization error between 12.7 to $14.4 \mathrm{~mm}$ (see table 2). While deviations of $10 \mathrm{~mm}$ are typically considered acceptable in a surgical setting, our error is a bit higher. Also, the tumor site was not detectable at realistic activity concentrations, while at higher activities it was visible with high localization errors. The errors are due to several reasons. Sub-optimal collimation in hand-held devices at high energies contributes to a decrease in accuracy. A remedy for this is to detect coincidences, which requires placing a detector block under the patient. On the other hand our system does not need such a complicated setup, and is thus a more feasible solution if it can meet the OR accuracy requirements. Although positron range in general affects PET image resolution [15, we do not think that it has a major effect on our system currently, due to the differences in the resolution range.

Some factors, however, can still be improved, like a better physical model of the detection process, taking also into account attenuation correction and scatter effects. The best solution to improve image quality would be to include registered prior PET/CT data, providing attenuation correction information as well as the possibility of guiding the scanning procedure and constraining the reconstruction process using priors. The combination with intra-operative ultrasound could for example yield the basis for registration of the prior data.

In summary, we have shown first results demonstrating feasibility of PET-like intra-operative imaging using $\mathrm{HE}$ probes within certain constraints with phantoms. In addition we have outlined the improvements to our method required for making it viable for clinical applications.

Acknowledgments. This research was funded/supported by the Graduate School of Information Science in Health (GSISH), the TUM Graduate School (Munich, Germany) and SFB 824 (DFG, Germany). 


\section{References}

1. Haddad, R.I., Shin, D.M.: Recent advances in head and neck cancer. The New England Journal of Medicine 359(11), 1143-1154 (2008)

2. Adams, S., Baum, R.P., Stuckensen, T., Bitter, K., Hör, G.: Prospective comparison of 18F-FDG PET with conventional imaging modalities (CT, MRI, US) in lymph node staging of head and neck cancer. Eur. J. Nucl. Med. 25(9), 1255-1260 (1998)

3. Wendler, T., Feuerstein, M., Traub, J., Lasser, T., Vogel, J., Daghighian, F., Ziegler, S.I., Navab, N.: Real-Time Fusion of Ultrasound and Gamma Probe for Navigated Localization of Liver Metastases. In: Ayache, N., Ourselin, S., Maeder, A. (eds.) MICCAI 2007, Part II. LNCS, vol. 4792, pp. 252-260. Springer, Heidelberg (2007)

4. Wendler, T., Herrmann, K., Schnelzer, A., Lasser, T., Traub, J., Kutter, O., Ehlerding, A., Scheidhauer, K., Schuster, T., Kiechle, M., Schwaiger, M., Navab, N., Ziegler, S.I., Buck, A.K.: First demonstration of 3-D lymphatic mapping in breast cancer using freehand SPECT. Eur. J. Nucl. Med. 37(8), 1452-1461 (2010)

5. Meller, B., Sommer, K., Gerl, J., von Hof, K., Surowiec, A., Richter, E., Wollenberg, B., Baehre, M.: High energy probe for detecting lymph node metastases with $18 \mathrm{~F}-$ FDG in patients with head and neck cancer. Nuklearmedizin 45(4), 153-159 (2006)

6. Kim, W.W., Kim, J.S., Hur, S.M., Kim, S.H., Lee, S., Choi, J.H., Kim, S., Choi, J.Y., Lee, J.E., Kim, J., Nam, S.J., Yang, J., Choe, J.: Radioguided surgery using an intraoperative PET probe for tumor localization and verification of complete resection in differentiated thyroid cancer: a pilot study. Surgery 149(3), 416-424 (2011)

7. Stolin, A.V., Majewski, S., Raylman, R.R., Martone, P.: Hand-Held SiPM-Based PET imagers for surgical applications. In: Proceedings of IEEE Nuclear Science Symposium and Medical Imaging Conference (IEEE NSS-MIC), Valencia, Spain (October 2011)

8. Majewski, S., Stolin, A., Martone, P., Raylman, R.: Dedicated mobile pet prostate imager. J. Nucl. Med. Meeting Abstracts 52(1), 1945 (2011)

9. Huber, J., Moses, W., Pouliot, J., Hsu, I.: Dual-Modality PET/Ultrasound imaging of the prostate, vol. 4, pp. 2187-2190. IEEE (October 2005)

10. Huh, S.S., Rogers, W.L., Clinthorne, N.H.: An investigation of an Intra-Operative PET imaging probe. In: Nuclear Science Symposium Conference Record, NSS 2007, pp. 552-555. IEEE (October 2007)

11. Huh, S.S., Rogers, W.L., Clinthorne, N.H.: On-line sliding-window list-mode PET image reconstruction for a surgical PET imaging probe. In: Nuclear Science Symposium Conference Record, NSS 2008, pp. 5479-5484. IEEE (October 2008)

12. Huh, S., Han, L., Rogers, W., Clinthorne, N.: Real time image reconstruction using GPUs for a surgical PET imaging probe system. In: Nuclear Science Symposium Conference Record, NSS 2009, pp. 4148-4153. IEEE (October 2009)

13. Cho, Z.H., Chan, J.K., Ericksson, L., Singh, M., Graham, S., MacDonald, N.S., Yano, Y.: Positron ranges obtained from biomedically important positron-emitting radionuclides. J. Nucl. Med. 16(12), 1174-1176 (1975)

14. National Institute of Standards and Technology, Physical Measurements Laboratory, http://physics.nist.gov/PhysRefData/Xcom/html/xcom1.html

15. Levin, C.S., Hoffman, E.J.: Calculation of positron range and its effect on the fundamental limit of positron emission tomography system spatial resolution. Physics in Medicine and Biology 44(3), 781-799 (1999) 